\title{
Overexpression of Wnt5a Promotes Angiogenesis in NSCLC
}

\author{
Lingli Yao, ${ }^{1,2}$ Baocun Sun,, ${ }^{1,3,4}$ Xiulan Zhao, ${ }^{1,4}$ Xueming Zhao, ${ }^{1}$ Qiang Gu, ${ }^{1,4}$ Xueyi Dong, \\ Yanjun Zheng, ${ }^{1}$ Junying Sun, ${ }^{1}$ Runfen Cheng, ${ }^{3}$ Hong Qi, ${ }^{1}$ and Jindan $\mathrm{An}^{3}$ \\ ${ }^{1}$ Department of Pathology, Tianjin Medical University, Tianjin 300070, China \\ ${ }^{2}$ Department of Pathology, Affiliated Anhui Provincial Hospital, Anhui Medical University, Hefei, Anhui 230001, China \\ ${ }^{3}$ Department of Pathology, Tianjin Cancer Hospital, Tianjin Medical University, Tianjin 300060, China \\ ${ }^{4}$ Department of Pathology, Tianjin General Hospital, Tianjin Medical University, Tianjin 300052, China
}

Correspondence should be addressed to Baocun Sun; baocunsun@gmail.com

Received 5 May 2014; Accepted 13 May 2014; Published 5 June 2014

Academic Editor: Shiwu Zhang

Copyright (C) 2014 Lingli Yao et al. This is an open access article distributed under the Creative Commons Attribution License, which permits unrestricted use, distribution, and reproduction in any medium, provided the original work is properly cited.

\begin{abstract}
To evaluate Wnt5a expression and its role in angiogenesis of non-small-cell lung cancer (NSCLC), immunohistochemistry and CD31/PAS double staining were performed to examine the Wnt5a expression and we analyze the relationships between Wnt5a and microvessel density (MVD), vasculogenic mimicry (VM), and some related proteins. About $61.95 \%$ of cases of 205 NSCLC specimens exhibited high expression of Wnt5a. Wnt5a expression level was upregulated in the majority of NSCLC tissues, especially in squamous cell carcinoma, while its expression level in adenocarcinoma was the lowest. Wnt5a was also found more frequently expressed in male patients than in female patients. Except for histological classification and gender, little association was found between Wnt5a and clinicopathological features. Moreover, Wnt5a was significantly correlated with prognosis. Overall, Wnt5apositive expression in patients with NSCLC indicated shorter survival time. As for vascularization in NSCLC, Wnt5a showed close association with VM and MVD. In addition, Wnt5a was positively related with $\beta$-catenin-nu, VE-cadherin, MMP2, and MMP9. The results demonstrated that overexpression of Wnt5a may play an important role in NSCLC angiogenesis and it may function via canonical Wnt signal pathway. This study will provide evidence for further research on NSCLC and also will provide new possible target for NSCLC diagnosis and therapeutic strategies.
\end{abstract}

\section{Introduction}

In recent years, primary lung cancer occupies the leading cause of cancer mortality in the world [1]. Among primary lung cancer cases, approximately $80 \%$ of the cases are nonsmall-cell lung cancer $[1,2]$. Although recent advancements have improved the survival rate of NSCLC patients [3-5], the high mortality related to NSCLC remains a daunting challenge [6]. Therefore, it is important to pay more attention to clarifying the mechanism of tumor biology in order to improve the prognosis of NSCLC patients. Indeed, angiogenesis theory has contributed significantly to tumor research.

Angiogenesis theory believes that tumor angiogenesis is essential for tumor growth and metastasis. When solid tumor grows to more than $2 \mathrm{~mm}$ in diameter, it needs to induce the generation new blood vessels to obtain a continuous supply of oxygen and nutrition to maintain its growth; otherwise, it would result in necrosis due to ischemia and anoxia $[7,8]$.
Thus, antiangiogenesis has become hot topic on tumor research. In fact, Wnt signaling pathway has been proven to be involved in this theory $[9,10]$.

Wnt5a is an important regulator of Wnt signaling pathway and has been demonstrated to play an important role in lung development and tumorigenesis [11, 12]. However, the biologics of Wnt5a in human cancers are still unclear. On the one hand, Wnt5a was found frequently upregulated in various cancers, including breast cancer, pancreatic cancer, prostate cancer, and gastric cancer [13-16]. On the other hand, Wnt5a was reported as a tumor suppressor gene in several cancers $[12,17,18]$. In addition, Wnt5a was proven to contribute to vascularization of embryonic stem cells [19]. Although Wnt5a was considered as an oncogene in lung cancer [20], its role in angiogenesis of lung cancer is still ambiguous.

Herein, the present study would investigate the expression of Wnt5a and its role in angiogenesis of human NSCLC. 
First, immunohistochemistry was performed to examine the Wnt5a expression in 205 NSCLC tissues. Then, the relationship between Wnt5a and microvessel density (MVD) was detected. In addition, the relationship between Wnt5a and vasculogenic mimicry (VM), a special blood supply mode, was also detected. Finally, correlations between Wnt5a and expressions of some angiogenesis-related proteins and $\beta$ catenin were analyzed.

\section{Materials and Methods}

2.1. Patients. Tissue specimens were obtained from 205 patients who had undergone surgical resection for lung cancer in Tianjin Medical University Cancer Institute and Hospital from October 1990 to November 2010. The 205 NSCLC samples were composed of 79 cases of squamous cell carcinoma, 75 cases of adenocarcinoma, and 51 cases of large cell lung cancer. The diagnoses of these samples were independently verified by two pathologists according to the standards of classification $[2,21]$. The average age of the patients at the time of diagnosis was 59.1 years (30 years to 88 years). The data of clinicopathological parameters were harvested from the patients' clinical records and pathological reports. Time to death, final follow-up examination, and diagnosis of metastasis were recorded from the date of surgery. This study was approved by the Ethical Committee of Tianjin Medical University prior to its initiation.

\subsection{Immunohistochemistry and CD31/Periodic Acid Schiff} (PAS) Double Staining. This assay was performed as described by Zhang et al. [22] and Sun et al. [23, 24]. The tissues were $10 \%$ formalin-fixed, paraffin-embedded, and cut into $4 \mu \mathrm{m}$ thickness. All slides were then deparaffinized in xylene and dehydrated with descending-grade alcohol. Endogenous peroxidase activity was quenched by brooding in methanol containing 3\% hydrogen peroxide for $30 \mathrm{~min}$ at room temperature. After blocking with recommended serum for $20 \mathrm{~min}$ at room temperature, the slides were incubated with a primary antibody overnight at $4^{\circ} \mathrm{C}$ and a homologous secondary antibody for $1 \mathrm{~h}$ at room temperature in a humidified box. Then the sections were stained with freshly dispensed diaminobenzidine solution (DAB) for observation under a microscope. In the process, the slides were all rinsed three times in phosphate-buffered saline (PBS) ( $\mathrm{pH}$ 7.2) before each step, except for the procedure of serum blocking to incubation with the primary antibody. The slides were then counterstained with hematoxylin, dehydrated with ascending-grade ethanol, air-dried, cleared with xylene, and mounted. For CD31/periodic acid Schiff (PAS) double staining, the sections were still incubated with $1 \%$ periodic acid for $15 \mathrm{~min}$ and Schiff reagent for observation under a microscope at $37^{\circ} \mathrm{C}$ between $\mathrm{DAB}$ staining and hematoxylin counterstaining. In this process, distilled water instead of PBS was used for washing.

In the current study, the primary antibodies to Wnt5a and VE-cadherin were purchased from Abcam (Cambridge, UK). Antibodies to CD31, CD34, $\beta$-catenin, and MMP2 were from Invitrogen Zymed Laboratories (San Diego, USA).
Antibody to MMP9 was from Santa Cruz Biotechnology (Santa Cruz, CA, USA). Positive control and negative control were performed for each batch. For the negative control, PBS was used instead of the primary antibody. For the positive control, a foregone positively expressed tissue section was used.

The results were evaluated following the method described by Bittner et al. [25]. The percentage and the intensity of the positive cells were both measured. The percentage was stratified as follows: 0 for less than $5 \%$ positive cells, 1 for less than $30 \%$ positive cells, 2 for less than $60 \%$ positive cells, and 3 for more than $60 \%$ positive cells. The intensity was also classified as follows: 0 (negative), 1 (weak), 2 (moderate), and 3 (strong). The sum of positive cell and staining intensity scores, which was more than 3 for the final result, was considered as the positive sample for each slide. Positive sample of $\beta$-catenin nuclear expression was deemed as those tissues which lost consecutive membrane location and acquired nuclear location more than $10 \%$ cells. MVD was determined from CD34-stained sections at the hot spot through light microscopy examination. The fields with the greatest neovascularization were examined by scanning tumor sections at low power $(\times 100)$. The average vessel count of the five fields $(\times 200)$ was regarded as the MVD.

2.3. Statistical Analysis. All data in the study were evaluated with SPSS17.0 software (SPSS, Chicago, IL, USA). Survival data were analyzed according to Kaplan-Meier test. Differences in survival curves were assessed by the log rank test. Crosstabs, Pearson $\chi^{2}$ test, and Spearman correlation analysis were used as needed. All $P$ values were two-sided, and $P<$ 0.05 was considered statistically significant.

\section{Results}

3.1. Association of Wnt5a with Clinicopathological Features in Human NSCLC. Wnt5a positive expression appeared as brown granules staining in the cytoplasms of the tumor cells. Among 205 NSCLC specimens, Wnt5a was detected in 127 cases (Figure 1). Approximately $61.95 \%$ of NSCLC exhibited high expression of Wnt5a. According to Wnt5a presence, all samples were divided into two groups: Wnt5a-positive group $(n=127)$ and Wnt5a-negative group $(n=78)$. Then, the relationship between $\mathrm{Wnt5}$ a and clinicopathological features was analyzed separately. Statistical data in Table 1 showed that Wnt5a was significantly associated with histological classification and gender $(P=0.016$ and 0.012 , resp.). Among the three histological types, Wnt5a was frequently expressed in squamous cell carcinoma (70.89\%, 56/79), while Wnt5a-positive expression in adenocarcinoma was the lowest (49.33\%, 37/75). In male samples, Wnt5a was found expressed more than in female patients $(67.59 \%, 98 / 145$ versus $48.33 \%$, 29/60). However, little correlation was found between Wnt5apositive expression and other clinicopathological characteristics, such as age, tumor size, location, histological differentiation, pleura invasion, stage, metastasis, lymph node status, and therapy before surgery $(P>0.05$, Table 1$)$. 


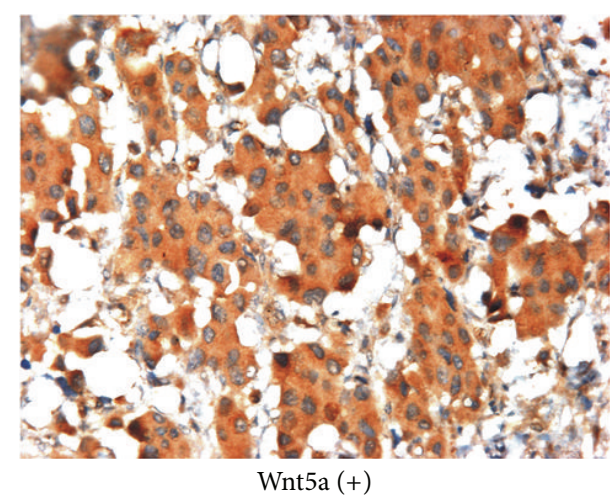

(a)

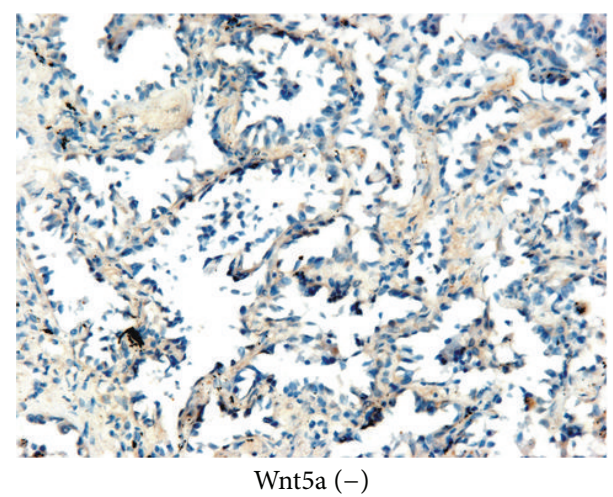

(b)

Figure 1: The expression of Wnt5a in NSCLC tissues (immunohistochemical staining, $\times 200$ ). Left photo showed that Wnt5a was positively expressed in the cytoplasm of tumor cells while Wnt5a was negatively expressed in the tumor cells at the right picture.

To verify the clinical significance of Wnt5a, all 205 NSCLC patients were followed up and the relationship between their outcomes and Wnt5a expression was examined. Statistical analysis showed that the overall survival period of Wnt5a-positive patients was enormously shorter than that of Wnt5a-negative patients $(P=0.026)$. The mean survival period of Wnt5a-negative group was 52.31 months, whereas that of Wnt5a-positive group was only 33.64 months (Figure 2).

3.2. Association of Wnt5a with Angiogenesis in Human NSCLC. To evaluate the role of Wnt5a in angiogenesis of NSCLC, relationships between MVD, VM, and Wnt5a were examined. CD34 was stained to calculate the MVD and CD31/PAS double staining was recruited to identify the VM (Figure 3). According to the median value of MVD or the presence of VM, all 205 NSCLC cases were classified as high CD34-MVD $(\geq 28, n=113)$ or low CD34-MVD $(<28, n=92)$ and divided into VM group $(n=28)$ or non-VM group $(n=177)$. As shown in Table 2 , significant correlation was found between Wnt5a and VM $(P=0.021, r=0.165)$, as well as Wnt5a and CD34-MVD $(P=0.026, r=0.157)$.

\subsection{Association of Wnt5a with Angiogenesis Related Proteins} in Human NSCLC. To further investigate the angiogenesis of NSCLC, some related proteins (VE-cadherin, MMP2, and MMP9) were also examined in this study. Positive expressions of these proteins were all located in the cytoplasm of tumor cells (Figure 4). Among 205 NSCLC tissues, VE-cadherin was positively expressed in 101 specimens $(49.27 \%, 101 / 205)$, while MMP2 and MMP9 were found positively expressed in 67 cases $(32.68 \%, 67 / 205)$ and 80 samples $(39.02 \%, 80 / 205)$, respectively. Moreover, the relationships between Wnt5a and these related proteins were also studied. VE-cadherin was found closely related with Wnt5a $(P=0.004, r=0.210)$ (Table 2). VE-cadherin-positive and Wnt5a-positive samples included 73 cases, and both negative samples included 50 cases. Similar to VE-cadherin, MMP2 and MMP9 both showed the remarkable relevance with Wnt5a $(P<0.001$, $r=0.268 ; P=0.003, r=0.215$, resp.) (Table 2).

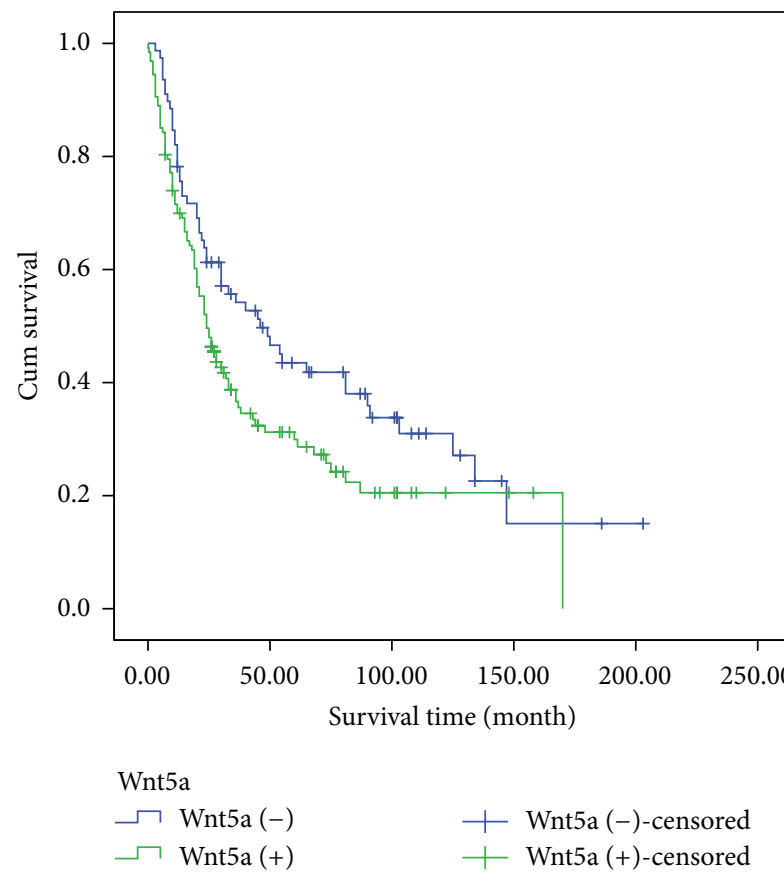

FIgURE 2: Result of the Kaplan-Meier survival analysis. KaplanMeier survival analysis showed that Wnt5a-positive patients have shorter survival periods than Wnt5a-negative patients $(P=0.026)$.

3.4. Association of Wnt5a with $\beta$-Catenin Nuclear Expression in Human NSCLC. To elucidate the possible mechanism of Wnt5a in angiogenesis of human NSCLC, $\beta$-catenin nuclear expression was detected and its relationship with Wnt5a was analyzed (Figure 5 ). In the current study, positive $\beta$-catenin nuclear expression appeared in 32 cases of 205 NSCLC tissues $(15.61 \%, 32 / 205)$. Wnt5a was shown to be closely associated with $\beta$-catenin nuclear location $(P=0.017$, $r=0.171$ ). Both Wnt5a and $\beta$-catenin nuclear locations were detected positively in 26 cases, while Wnt5a and $\beta$ catenin nuclear locations were both negatively expressed in 72 samples. Wnt5a-positive expression but $\beta$-catenin-nu 
TABLE 1: Correlation between Wnt5a and clinicopathological features in NSCLC.

\begin{tabular}{cccccc}
\hline \multirow{2}{*}{ Variant } & Total & \multicolumn{3}{c}{ Wnt5a } & \\
& $n$ & $\begin{array}{c}\text { Negative } \\
(\%)\end{array}$ & Positive & $x^{2}$ & $P$ value \\
& & $\%)$ & & \\
\hline
\end{tabular}

\begin{tabular}{lccccc}
\hline Gender & & & & & \\
Male & 145 & $47(32.41)$ & $98(67.59)$ & 6.674 & 0.012 \\
Female & 60 & $31(51.67)$ & $29(48.33)$ & & \\
Age (yr) & & & & & \\
$<60$ & 101 & $37(36.63)$ & $64(63.37)$ & 0.169 & 0.774 \\
$\geq 60$ & 104 & $41(39.42)$ & $63(60.58)$ & &
\end{tabular}

Size $(\mathrm{cm})$

$\begin{array}{lccccc}<3 & 29 & 11(37.93) & 18(62.07) & 0.000 & 1.000 \\ \geq 3 & 176 & 67(38.07) & 109(61.93) & & \end{array}$

Location

$\begin{array}{lccccc}\text { Center } & 106 & 38(35.85) & 68(64.15) & 0.451 & 0.565 \\ \text { Peripheral } & 99 & 40(40.40) & 59(59.60) & & \end{array}$

Histological

classification

$\begin{array}{llllll}\text { SCC } & 79 & 23(29.11) & 56(70.89) & & \\ \text { AC } & 75 & 38(50.67) & 37(49.33) & 8.222 & 0.016 \\ \text { LCC } & 51 & 17(33.33) & 34(66.67) & & \\ \text { Differentiation } & & & & & \\ \text { Well } & 35 & 17(48.57) & 18(51.43) & & \\ \text { Moderate } & 87 & 32(36.78) & 55(63.22) & 2.044 & 0.360 \\ \text { Poor } & 83 & 29(34.94) & 54(65.06) & & \\ \text { Pleura invasion } & & & & & \\ \text { No } & 113 & 39(34.51) & 74(65.49) & 1.335 & 0.252 \\ \text { Yes } & 92 & 39(42.39) & 53(57.61) & & \end{array}$

Lymph node

metastasis

$\begin{array}{llllll}\text { No } & 117 & 45(38.46) & 72(61.54) & 0.020 & 1.000 \\ \text { Yes } & 88 & 33(37.50) & 55(62.50) & & \end{array}$

T stage

$\begin{array}{cccccc}\text { T1 + T2 } & 149 & 56(37.58) & 93(62.42) & 0.050 & 0.872 \\ \text { T3 + T4 } & 56 & 22(39.29) & 34(60.71) & & \\ \text { Clinical stage } & & & & & \\ \text { I + II } & 158 & 62(39.24) & 96(60.76) & 0.415 & 0.609 \\ \text { III + IV } & 47 & 16(34.04) & 31(65.96) & & \end{array}$

Distant

metastasis

$\begin{array}{lrrrrr}\text { No } & 147 & 57(38.78) & 90(61.22) & 0.116 & 0.752 \\ \text { Yes } & 58 & 21(36.21) & 37(63.79) & & \end{array}$

Therapy before

surgery

\begin{tabular}{cccccc} 
No & 186 & $69(37.10)$ & $117(62.90)$ & 0.772 & 0.458 \\
Yes & 19 & $9(47.37)$ & $10(52.63)$ & & \\
\hline
\end{tabular}

$P<0.05$ means statistical significance.

negative location was found in 101 tissues, whereas $\beta$-cateninnu positive and Wnt5a-negative samples included 6 cases (Table 2).
TABLE 2: Relationship between Wnt5a and angiogenesis, expression of related proteins, and $\beta$-catenin nuclear expression in NSCLC.

\begin{tabular}{|c|c|c|c|c|c|}
\hline \multirow{2}{*}{ Variant } & \multirow{2}{*}{$\begin{array}{c}\text { Total } \\
n\end{array}$} & \multicolumn{2}{|c|}{ Wnt5a } & \multirow{2}{*}{$P$ value } & \multirow{2}{*}{$r$} \\
\hline & & Negative (\%) & Positive (\%) & & \\
\hline \multicolumn{6}{|l|}{ CD34-MVD } \\
\hline$<28$ & 92 & $52(56.52)$ & $40(43.48)$ & \multirow{2}{*}{0.026} & \multirow{2}{*}{0.157} \\
\hline$\geq 28$ & 113 & $46(40.71)$ & 67 (59.29) & & \\
\hline \multicolumn{6}{|l|}{ VM } \\
\hline Negative & 177 & $73(41.24)$ & $104(58.76)$ & \multirow{2}{*}{0.021} & \multirow{2}{*}{0.165} \\
\hline Positive & 28 & $5(17.86)$ & $23(82.14)$ & & \\
\hline \multicolumn{6}{|l|}{ VE-cadherin } \\
\hline Negative & 104 & $50(48.08)$ & $54(51.92)$ & \multirow{2}{*}{0.004} & \multirow{2}{*}{0.210} \\
\hline Positive & 101 & $28(27.72)$ & $73(72.28)$ & & \\
\hline \multicolumn{6}{|l|}{ MMP2 } \\
\hline Negative & 138 & $65(47.10)$ & $73(52.90)$ & \multirow{2}{*}{$<0.001$} & \multirow{2}{*}{0.268} \\
\hline Positive & 67 & $13(19.40)$ & $54(80.60)$ & & \\
\hline \multicolumn{6}{|l|}{ MMP9 } \\
\hline Negative & 125 & $58(46.40)$ & $67(53.60)$ & \multirow{2}{*}{0.003} & \multirow{2}{*}{0.215} \\
\hline Positive & 80 & $20(25.00)$ & $60(75.00)$ & & \\
\hline \multicolumn{6}{|l|}{$\begin{array}{l}\beta \text {-Catenin } \\
\text { nuclear } \\
\text { expression }\end{array}$} \\
\hline Negative & 173 & $72(41.62)$ & $101(58.38)$ & \multirow{2}{*}{0.017} & \multirow{2}{*}{0.171} \\
\hline Positive & 32 & $6(18.75)$ & $26(81.25)$ & & \\
\hline
\end{tabular}

\section{Discussion}

The Wnt proteins family includes at least 19 secreted cysteinerich glycoproteins that are involved in the regulation of a wide variety of normal and pathologic processes, including embryogenesis, differentiation, and tumorigenesis [26-30]. As one of the important members in the large Wnt family, Wnt5a has been shown to have close correlation with various cancers $[13-15,18]$.

In this study, expression of Wnt5a was investigated by immunohistochemistry in a large cohort of 205 human NSCLC tissues. The results showed that Wnt5a was upexpressed in the majority of cases. Except for histological classification and gender, Wnt5a-positive expression was found to exhibit little correlation with clinicopathological parameters. Wnt5a was more frequently expressed in squamous cell carcinoma. Our result was in accordance with the previous report. Huang and his colleagues examined Wnt5a expression in 123 NSCLC cases and found a similar result [20]. We also found that Wnt5a was more often expressed in male NSCLC patients, although none of the previous studies examined the relation between Wnt5a and gender in NSCLC $[20,31]$. However, two studies strongly supported our result. Heikkila et al. reported that deficiency of Wnt5a could result in sex reversal, infertility, and/or malformation of the internal and external genitals [32]. In addition, Kovalchuk et al. concluded that Wnt signaling pathway, including Wnt5a, was differently induced between male and female mice after 


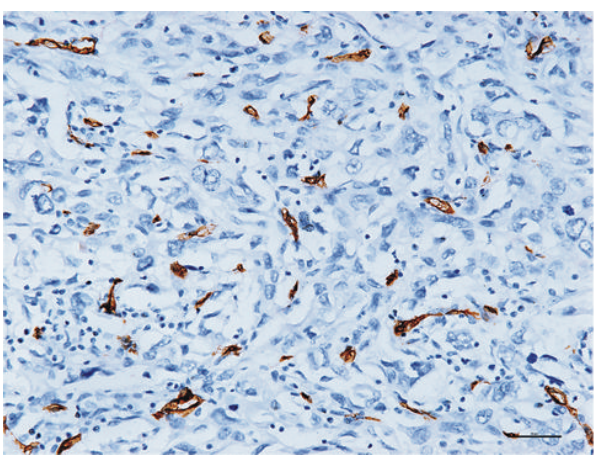

(a)

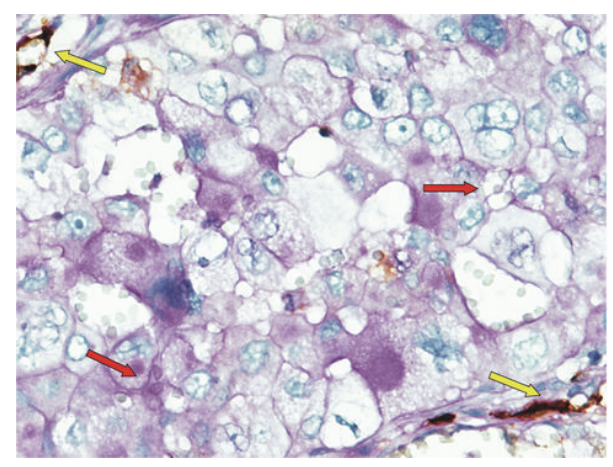

(b)

FIgURE 3: The angiogenesis status in NSCLC. (a) MVD staining for CD34 in NSCLC (immunohistochemical staining, $\times 200$ ). A hotspot with high MVD was positively stained. (b) CD31/PAS double staining for VM $(\times 400)$. The VM channel showed a positive expression for PAS but a negative expression for CD31 (red arrow). The endothelial channel showed positive expressions for both CD31 and PAS (yellow arrow).
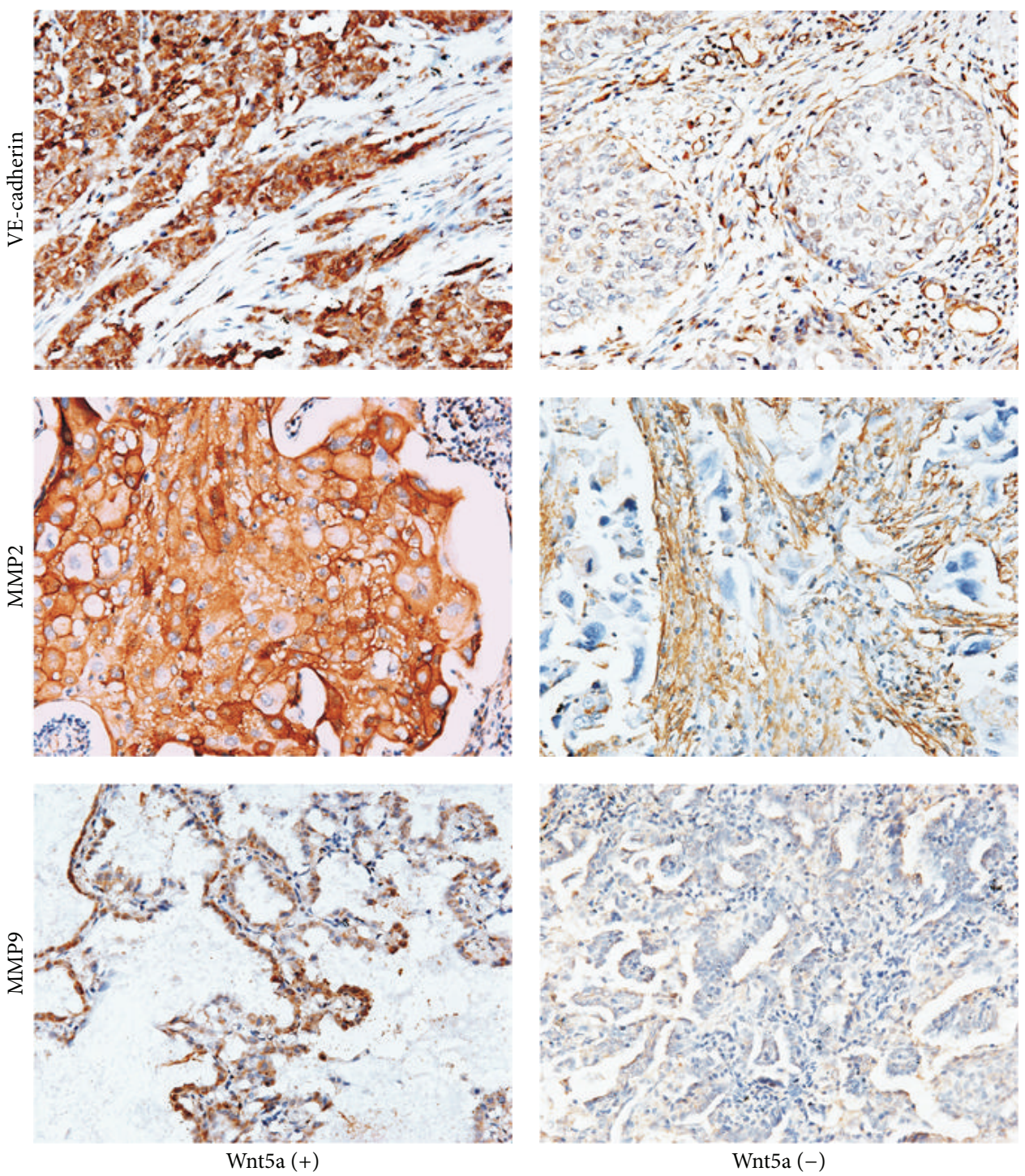

FIGURE 4: The expressions of some related proteins in NSCLC (immunohistochemical staining, $\times 200$ ). In Wnt5a-positive group, positive expressions of VE-cadherin, MMP2, and MMP9 were all located in the cytoplasm of tumor cells. However, these proteins were negatively expressed in NSCLC tissues of Wnt5a-negative group. VE-cadherin positive expression in endothelial cells or MMPs positive expression in stromal cells could provide an internal positive control for both, respectively. 

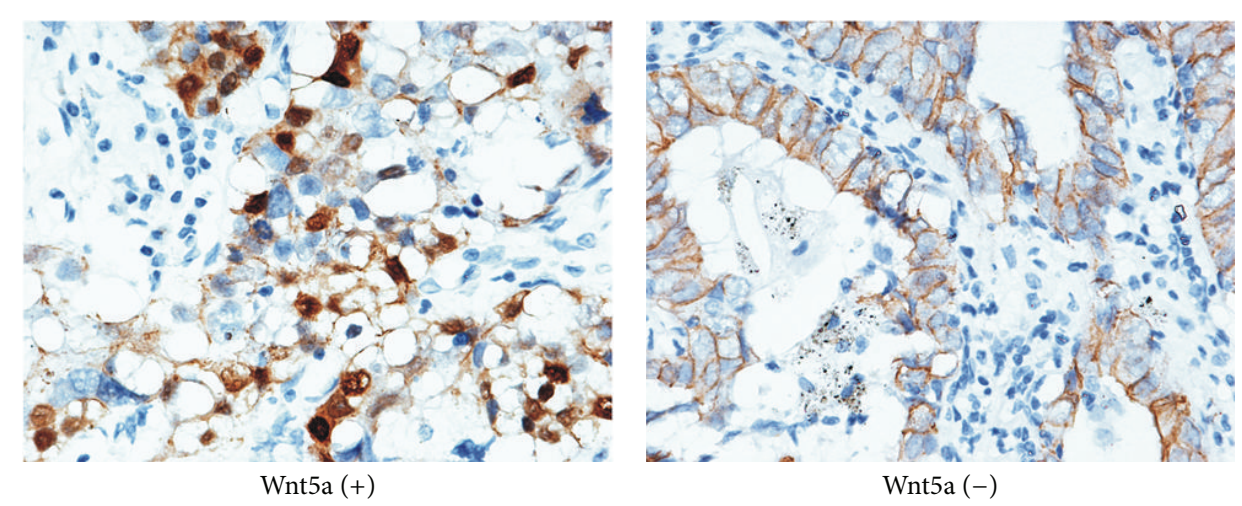

Figure 5: The expression of $\beta$-catenin in NSCLC (immunohistochemical staining, $\times 400$ ). In Wnt5a-negative tissues, $\beta$-catenin exhibited the continuous expression in membrane, while in Wnt5a-positive group, $\beta$-catenin lost its continuous membrane expression and translocated to nucleus.

chronic radiation exposure [33]. Thus, we supposed that it may be related with sex hormone. Moreover, our data showed that overall survival time of those NSCLC patients with Wnt5a-positive expression was shorter than that of Wnt5a negative-expressed patients, which supported the results of Huang et al. and Nakashima et al. [20, 31]. Therefore, it may provide a good marker for clinical diagnosis and prognosis of NSCLC.

It is well known that a continuous supply of oxygen and nutrition is crucial for indefinite growth of solid tumors. Angiogenesis plays a vital role in development of tumor. Huang et al. investigated the relationship between Wnt5a and angiogenesis in 123 NSCLCs which included 67 cases of adenocarcinoma (AC), 50 cases of squamous cell carcinoma (SCC), and 6 cases of large cell carcinoma (LCC). They reported that the intratumoral Wnt5a expression was significantly correlated with the stromal expression of VEGFA but insignificantly correlated with intratumoral microvessel density [20]. In this study, we also examined the relationship between Wnt5a and angiogenesis in NSCLC. Firstly, we evaluated the relation between Wnt5a and MVD, which is a classic marker of tumor angiogenesis. However, contrary to previous results [20], we found that there was significant correlation between Wnt5a and MVD in the present study. We supposed that it is due to the difference of object. Our study contained more samples including 205 NSCLC specimens. Moreover, the ratio for LCC was larger than Huang's study. As we know, LCC is a more progressive tumor in NSCLC. In addition, they only reported the relationship between Wnt5a and MVD, which only reflected the endothelial dependent vessel in the tumor. However, besides endothelial dependent vessel, Maniotis detected a novel blood supply mode, named vasculogenic mimicry (VM), in highly aggressive uveal melanomas [34]. Subsequently, many researchers have found VM in several malignant tumors [22, 35-40]. Thus, we also analyzed the correlation between Wnt5a and VM, which could reflect the blood supply of tumor at some degree. Statistical analysis showed that Wnt5a was positively correlated with VM. Wnt5a may play an important role in angiogenesis of NSCLC.

To further investigate the relationship between Wnt5a and angiogenesis, we conducted immunohistochemistry to examine expressions of some related proteins and analyzed their associations with Wnt5a. VE-cadherin, MMP2, and MMP9 have been proven to participate and represent partially VM [35, 39, 41]. Our data showed that Wnt5a was also positively associated with all the above proteins. The results further demonstrated that Wnt5a play an active role in angiogenesis of NSCLC.

We also detected $\beta$-catenin nuclear expression in NSCLC tissues and found it was positively correlated with Wnt5a. $\beta$ Catenin is a vital molecule of canonical Wnt signal pathway. Normally, $\beta$-catenin shows continuous membrane location, and while canonical Wnt pathway is activated, $\beta$-catenin shows translocation to nucleus. Thus, $\beta$-catenin nuclear expression is considered as an important marker for activated canonical Wnt pathway. Therefore, our data indicated that Wnt5a may function through canonical Wnt pathway. Though it was a member of noncanonical Wnt pathway, Wnt5a was reported to play a role in the canonical Wnt $/ \beta$ catenin signaling pathway. The Wnt5a protein can act via Frz5 receptor to initiate an intracellular pathway leading to the accumulation of $\beta$-catenin $[20,42]$.

\section{Conclusions}

Taken together, the current study demonstrated that Wnt5a was overexpressed in human NSCLC tissues and closely associated with tumor angiogenesis. Enhanced expression of Wnt5a may induce the nuclear accumulation of $\beta$-catenin and activate the canonical Wnt signaling pathway, thus leading to the upexpression of VE-cadherin, MMP2, and MMP9, then resulting in angiogenesis, and ultimately promoting the growth and metastasis of NSCLC. Therefore, this study may contribute to the mechanism of NSCLC research and provide new hope for NSCLC diagnosis and therapeutic strategies.

\section{Conflict of Interests}

The authors declare that there is no conflict of interests regarding the publication of this paper. 


\section{Authors' Contribution}

The conception and design of the study, acquisition of data, most of immunohistochemical staining, analysis and interpretation of data, and drafting of the paper were performed by Lingli Yao. Baocun Sun and Xiulan Zhao made the decision of the conception and design of the study. Professor Sun is also responsible for this paper. All $4 \mu \mathrm{m}$ slides, $\mathrm{H} \& \mathrm{E}$ staining, and CD31/PAS staining were performed by Xueming Zhao, Qiang Gu, and Xueyi Dong. Some supporting works, such as followup and collecting patients' records, were accomplished by Yanjun Zheng, Junying Sun, and Jindan An. A small part of immunohistochemical staining was performed by Runfen Cheng and Hong Qi. Lingli Yao, Baocun Sun, and Xiulan Zhao equally contributed to this study.

\section{Acknowledgments}

This work was supported by Grants from the Key project of the National Natural Science Foundation of China (no. 81230050), the National Natural Science Foundation of China (nos. 81172046 and 81173091), and the Key Project of the Tianjin Natural Science Foundation (no. 12JCZDJC23600).

\section{References}

[1] R. Siegel, J. Ma, Z. Zou, and A. Jemal, "Cancer statistics, 2014," CA: A Cancer Journal for Clinicians, vol. 64, pp. 9-29, 2014.

[2] M. B. Beasley, E. Brambilla, and W. D. Travis, "The 2004 World Health Organization classification of lung tumors," Seminars in Roentgenology, vol. 40, no. 2, pp. 90-97, 2005.

[3] L. Tao, G. Huang, S. Shi, and L. Chen, "Bevacizumab improves the antitumor efficacy of adoptive cytokine-induced killer cells therapy in non-small cell lung cancer models," Medical Oncology, vol. 31, p. 777, 2014.

[4] A. Pircher, I. K. Wasle, M. Mian et al., "Docetaxel in the treatment of non-small cell lung cancer (NSCLC) - an observational study focusing on symptom improvement," Anticancer Research, vol. 33, pp. 3831-3836, 2013.

[5] M. Christodoulou, N. Bayman, P. McCloskey, C. Rowbottom, and C. Faivre-Finn, "New radiotherapy approaches in locally advanced non-small cell lung cancer," European Journal of Cancer, vol. 50, pp. 525-534, 2014.

[6] V. L. Keedy, S. Temin, M. R. Somerfield et al., "American Society of clinical oncology provisional clinical opinion: epidermal growth factor receptor (EGFR) mutation testing for patients with advanced non-small-cell lung cancer considering firstline EGFR tyrosine kinase inhibitor therapy," Journal of Clinical Oncology, vol. 29, no. 15, pp. 2121-2127, 2011.

[7] J. Folkman, "Tumor angiogenesis: therapeutic implications," The New England Journal of Medicine, vol. 285, no. 21, pp. 1182$1186,1971$.

[8] J. Folkman, "Angiogenesis in cancer, vascular, rheumatoid and other disease," Nature Medicine, vol. 1, no. 1, pp. 27-31, 1995.

[9] H. Park, H. Y. Jung, H. J. Choi et al., "Distinct roles of DKK1 and DKK2 in tumor angiogenesis," Angiogenesis, vol. 17, pp. 221-234, 2014.

[10] J. A. Stefater III, I. Lewkowich, S. Rao et al., "Regulation of angiogenesis by a non-canonical Wnt-Flt1 pathway in myeloid cells," Nature, vol. 474, no. 7352, pp. 511-516, 2011.
[11] T. N. H. Masckauchán, D. Agalliu, M. Vorontchikhina et al., "Wnt5a signaling induces proliferation and survival of endothelial cells in vitro and expression of MMP-1 and Tie-2," Molecular Biology of the Cell, vol. 17, no. 12, pp. 5163-5172, 2006.

[12] R. Cheng, B. Sun, Z. Liu et al., "Wnt5a suppresses colon cancer by inhibiting cell proliferation and epithelial-mesenchymal transition," Journal of Cellular Physiology, 2014.

[13] M. Fernandez-Cobo, F. Zammarchi, J. Mandeli, J. F. Holland, and B. G. T. Pogo, "Expression of Wnt5A and Wnt10B in nonimmortalized breast cancer cells," Oncology Reports, vol. 17, no. 4, pp. 903-907, 2007.

[14] H. Bo, S. Zhang, L. Gao et al., "Upregulation of Wnt5a promotes epithelial-to-mesenchymal transition and metastasis of pancreatic cancer cells," BMC Cancer, vol. 13, article 496, 2013.

[15] G. T. Lee, D. I. Kang, Y. S. Ha et al., "Prostate cancer bone metastases acquire resistance to androgen deprivation via WNT5Amediated BMP-6 induction," The British Journal of Cancer, vol. 110, pp. 1634-1644, 2014.

[16] M. Kanzawa, S. Semba, S. Hara, T. Itoh, and H. Yokozaki, "WNT5A is a key regulator of the epithelial-mesenchymal transition and cancer stem cell properties in human gastric carcinoma cells," Pathobiology, vol. 80, no. 5, pp. 235-244, 2013.

[17] W. Jiang, D. K. Crossman, E. H. Mitchell, P. Sohn, M. R. Crowley, and R. Serra, "WNT5A inhibits metastasis and alters splicing of Cd44 in breast cancer cells," PLoS ONE, vol. 8, no. 3, Article ID e58329, 2013.

[18] L. Bi, X. Liu, C. Wang et al., "Wnt5a involved in regulation of the biological behavior of hepatocellular carcinoma," International Journal of Clinical and Experimental Pathology, vol. 7, pp. 987995, 2014.

[19] D.-H. Yang, J.-Y. Yoon, S.-H. Lee et al., "Wnt5a is required for endothelial differentiation of embryonic stem cells and vascularization via pathways involving both $\mathrm{Wnt} / \beta$-Catenin and protein kinase C $\alpha$," Circulation Research, vol. 104, no. 3, pp. 372-379, 2009.

[20] C.-L. Huang, D. Liu, J. Nakano et al., "Wnt5a expression is associated with the tumor proliferation and the stromal vascular endothelial growth factor-an expression in non-small-cell lung cancer," Journal of Clinical Oncology, vol. 23, no. 34, pp. 8765-8773, 2005.

[21] W. D. Travis, E. Brambilla, M. Noguchi et al., "International association for the study of lung cancer/American Thoracic Society/European Respiratory Society international multidisciplinary classification of lung adenocarcinoma," Journal of Thoracic Oncology, vol. 6, no. 2, pp. 244-285, 2011.

[22] Y. Zhang, B. Sun, X. Zhao et al., "Clinical significances and prognostic value of cancer stem-like cells markers and vasculogenic mimicry in renal cell carcinoma," Journal of Surgical Oncology, vol. 108, no. 6, pp. 414-419, 2013.

[23] T. Sun, N. Zhao, X.-L. Zhao et al., "Expression and functional significance of Twistl in hepatocellular carcinoma: its role in vasculogenic mimicry," Hepatology, vol. 51, no. 2, pp. 545-556, 2010.

[24] T. Sun, B.-C. Sun, X.-L. Zhao et al., "Promotion of tumor cell metastasis and vasculogenic mimicry by way of transcription coactivation by Bcl-2 and Twist1: a study of hepatocellular carcinoma," Hepatology, vol. 54, no. 5, pp. 1690-1706, 2011.

[25] M. Bittner, P. Meltzer, Y. Chen et al., "Molecular classification of cutaneous malignant melanoma by gene expression profiling," Nature, vol. 406, no. 6795, pp. 536-540, 2000. 
[26] C. Lange, E. Mix, K. Rateitschak, and A. Rolfs, "Wnt signal pathways and neural stem cell differentiation," Neurodegenerative Diseases, vol. 3, no. 1-2, pp. 76-86, 2006.

[27] D. Herzlinger, J. Qiao, D. Cohen, N. Ramakrishna, and A. M. C. Brown, "Induction of kidney epithelial morphogenesis by cells expressing Wnt-1," Developmental Biology, vol. 166, no. 2, pp. 815-818, 1994.

[28] T. C. Dale, "Signal transduction by the Wnt family of ligands," Biochemical Journal, vol. 329, no. 2, pp. 209-223, 1998.

[29] Z. You, D. Saims, S. Chen et al., "Wnt signaling promotes oncogenic transformation by inhibiting c-Myc-induced apoptosis," Journal of Cell Biology, vol. 157, no. 3, pp. 429-440, 2002.

[30] T. Reya and H. Clevers, "Wnt signalling in stem cells and cancer," Nature, vol. 434, no. 7035, pp. 843-850, 2005.

[31] N. Nakashima, C.-L. Huang, D. Liu, M. Ueno, and H. Yokomise, "Intratumoral Wntl expression affects survivin gene expression in non-small cell lung cancer," International Journal of Oncology, vol. 37, no. 3, pp. 687-694, 2010.

[32] M. Heikkil, H. Peltoketo, and S. Vainio, "Wnts and the female reproductive system," Journal of Experimental Zoology, vol. 290, no. 6, pp. 616-623, 2001.

[33] O. Kovalchuk, A. Ponton, J. Filkowski, and I. Kovalchuk, "Dissimilar genome response to acute and chronic low-dose radiation in male and female mice," Mutation Research, vol. 550, no. 1-2, pp. 59-72, 2004.

[34] A. J. Maniotis, R. Folberg, A. Hess et al., "Vascular channel formation by human melanoma cells in vivo and in vitro: vasculogenic mimicry," The American Journal of Pathology, vol. 155, no. 3, pp. 739-752, 1999.

[35] J. Du, B. Sun, X. Zhao et al., "Hypoxia promotes vasculogenic mimicry formation by inducing epithelial-mesenchymal transition in ovarian carcinoma," Gynecologic Oncology, 2014.

[36] H. J. Chung and M. Mahalingam, "Angiogenesis, vasculogenic mimicry and vascular invasion in cutaneous malignant melanoma-implications for therapeutic strategies and targeted therapies," Expert Review of Anticancer Therapy, vol. 14, no. 5, pp. 621-639, 2014.

[37] J. T. Zhang, W. Sun, W. Z. Zhang et al., "Norcantharidin inhibits tumor growth and vasculogenic mimicry of human gallbladder carcinomas by suppression of the PI3-K/MMPs/Ln-5gamma2 signaling pathway," BMC Cancer, vol. 14, article 193, 2014.

[38] F. Luo, K. Yang, R. L. Liu, C. Meng, R. F. Dang, and Y. Xu, "Formation of vasculogenic mimicry in bone metastasis of prostate cancer: correlation with cell apoptosis and senescence regulation pathways," Pathology: Research and Practice, vol. 210, pp. 291-295, 2014.

[39] K. Chiablaem, K. Lirdprapamongkol, S. Keeratichamroen, R. Surarit, and J. Svasti, "Curcumin suppresses vasculogenic mimicry capacity of hepatocellular carcinoma cells through STAT3 and PI3K/AKT inhibition," Anticancer Research, vol. 34, pp. 1857-1864, 2014.

[40] Y. S. Chen and Z. P. Chen, "Vasculogenic mimicry: a novel target for glioma therapy," Chinese Journal of Cancer, vol. 33, pp. 74-79, 2014.

[41] W.-B. Liu, G.-L. Xu, W.-D. Jia et al., "Prognostic significance and mechanisms of patterned matrix vasculogenic mimicry in hepatocellular carcinoma," Medical Oncology, vol. 28, supplement 1, pp. S228-S238, 2011.

[42] X. He, J.-P. Saint-Jeannet, Y. Wang, J. Nathans, I. Dawid, and H. Varmus, "A member of the frizzled protein family mediating axis induction by Wnt-5A," Science, vol. 275, no. 5306, pp. 1652$1654,1997$. 


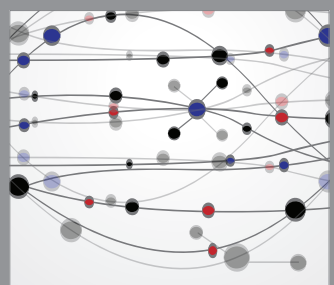

The Scientific World Journal
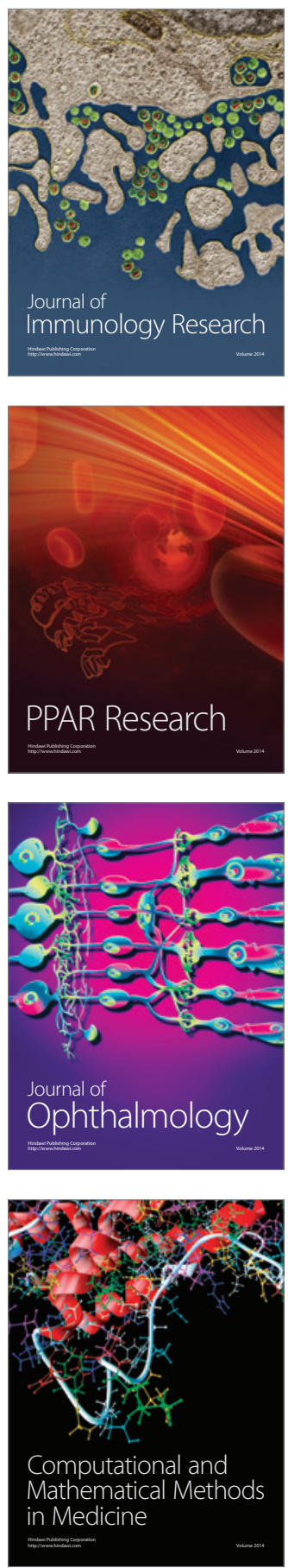

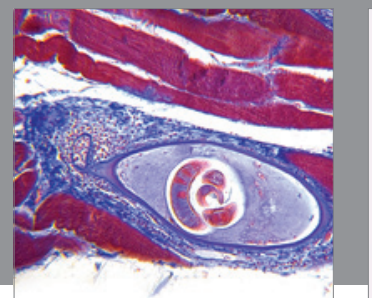

Gastroenterology

Research and Practice
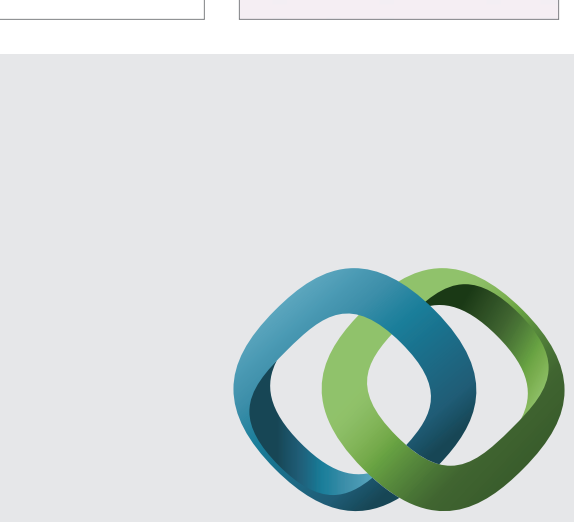

\section{Hindawi}

Submit your manuscripts at

http://www.hindawi.com
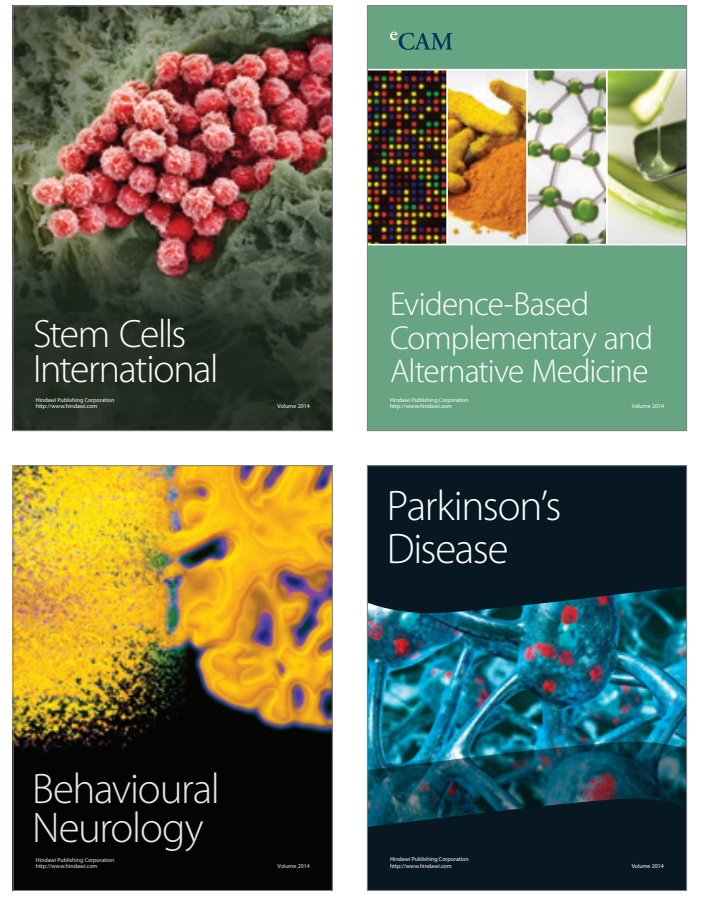
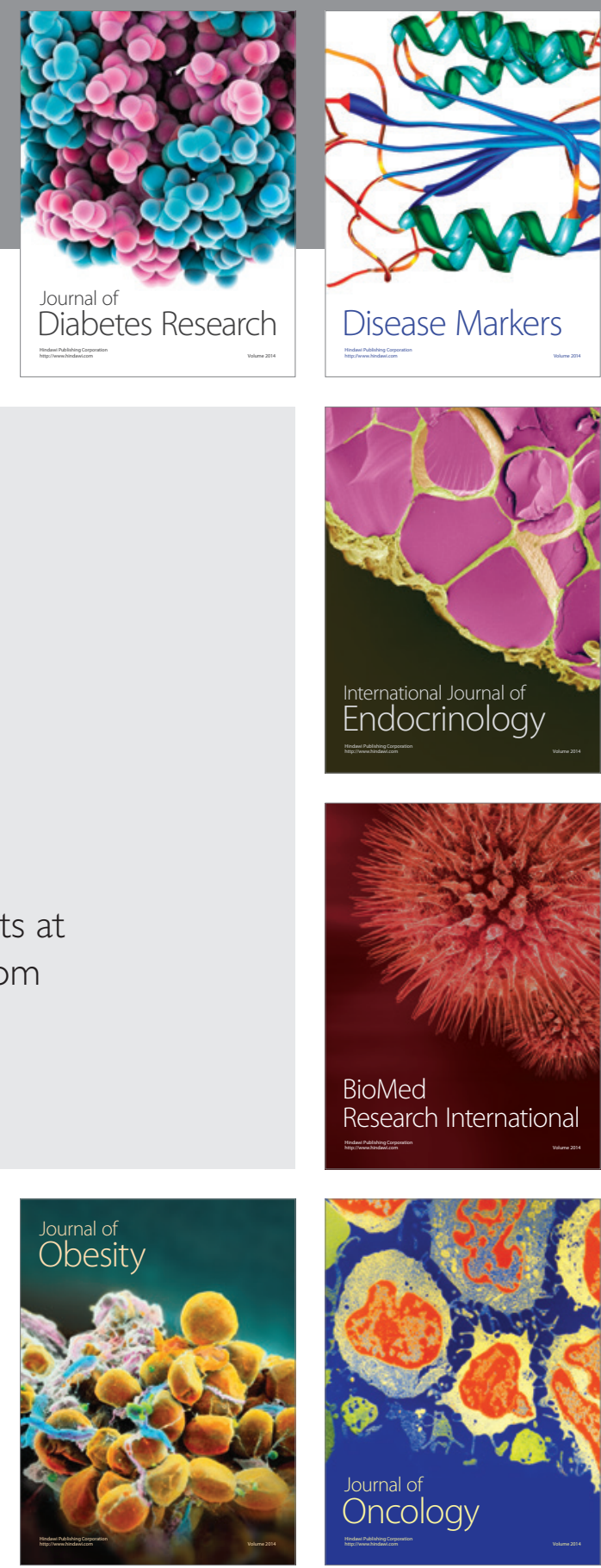

Disease Markers
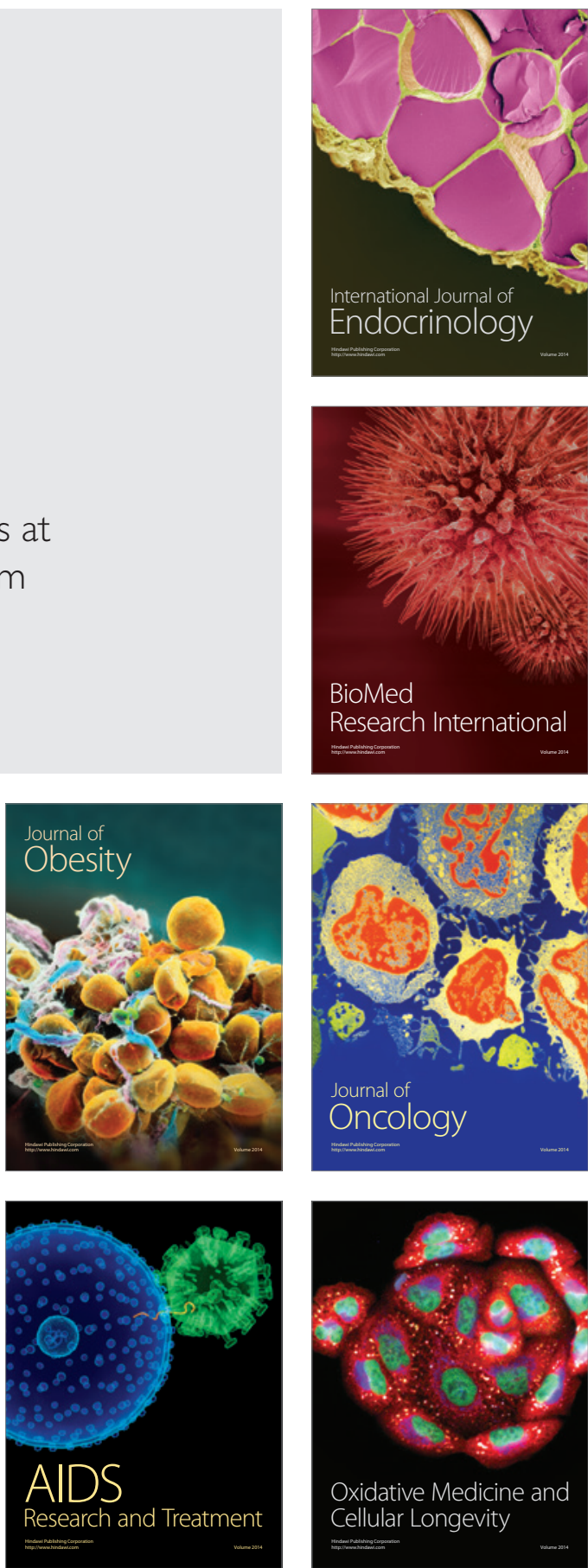